\title{
La parte negada del parto institucionalizado: explorando sus bases antropológicas
}

\author{
Denied Part of Institutionalized Delivery: \\ Exploring Their Anthropological Bases
}

\author{
José Manuel Hernández Garre \\ Paloma Echevarría Pérez \\ Universidad Católica de Murcia
}

\section{RESUMEN}

El objetivo del artículo ha sido explorar, a través de un exhaustivo análisis documental, las características y bases antropológicas del parto institucionalizado. Los resultados muestran cómo los nacimientos hospitalarios se han convertido en un ritual clínico cuyo fin es controlar la procreación a través del uso indiscriminado de la tecnología y las nociones tayloristas. Las madres se han transformado en sujetos pasivos de sus propios partos, mientras la injerencia anatómica y el control biométrico de su dimensión corporal se convertían en norma. Se trata, en definitiva, de un auténtico patrón cultural de asistencia, caracterizado por la medicalización, el control biomecánico y la mecanización del proceso, atributos alimentados, a su vez, por toda una serie de ideologías y bases antropológicas de fondo como la biopolítica, el posthumanismo o el productivismo. Se concluye, pues, que la institucionalización de los partos en Occidente se ha debido más a causas culturales e ideológicas que a la supuesta practicidad epidemiológica defendida desde las ciencias médicas.

Palabras clave: Parto institucionalizado, Parto hospitalario, Parto medicalizado, Procreación.

\section{SUMMARY}

The objective of this article was to explore, through a comprehensive documentary analysis, features and anthropological bases of institutionalized delivery. The results show as hospital births have become a clinical ritual whose purpose is to control procreation through the indiscriminate use of technology and taylorist notions. Mothers have become passive subjects of their own births, while the interference anatomic and biometric control of their body became standard. It is, in short, a true cultural attendance pattern characterized by medicalization, biomechanical control and mechanization of the process, attributes fed, in turn, by a range of ideologies and anthropological bases background as biopolitics, the posthumanism or productivism. It is therefore concluded that the institutionalization of deliveries in the West has been due more to cultural and ideological causes than the alleged practicality epidemiological defended from medical science.

Key words: Institutionalized childbirth, Hospitalized birth, Medicalized birth, Procreation. 


\section{INTRODUCCIÓN}

La obsesión por el control estatal sobre la propia fertilidad, con el fin de conseguir ciudadanos productivos, ha contaminado los pilares mismos de la existencia, propiciando la descontextualización de los nacimientos de los domicilios, para ser traslados a las frías instituciones hospitalarias. Esta migración del parto, producida desde finales de la Segunda Guerra Mundial, se ha debido al aura de misterio que ha rodeado a la atención especializada dada por los médicos en el seno de los hospitales, y a la falsa idea de que los hospitales eran centros libres de bacterias, a pesar de que las infecciones obstétricas se presentaran también en el seno de los mismos (Vélez et al. 2005). La búsqueda utópica del riesgo cero ha provocado una auténtica revolución en el modo de dar a luz, transformándose el rol de las parturientas y los profesionales implicados en el proceso (Towler y Bramall 1997), hasta el punto de que en determinados contextos "el bienestar y los deseos de los individuos se han sacrificado gradualmente en aras de los intereses burocráticos y profesionales, adquiriendo la tecnología más importancia que las necesidades de los individuos" (Huntingford 1978: 8). Por primera vez los alumbramientos corren el riesgo de no ser algo que hacen las mujeres, que simplemente acontece, sino algo que los especialistas les hacen a las mujeres, algo provocado, inducido (Tweedie 1974). Las razones argüidas desde la disciplina que ostenta el poder estatal sobre la dimensión corporal del ser humano, la biomedicina, se reducen a la defensa de que esta reclusión del parto en el hospital ha reducido espectacularmente las cifras de mortalidad ${ }^{1}$. El supuesto pragmatismo prevalece por tanto sobre la experiencia desde los mismos albores de la existencia, imponiéndose la mecanización fabril de los alumbramientos a la vivencia espontánea.

No se trata de un tema trivial, ya que están en juego los engranajes que dictan la forma en que se aborda el principio de nuestra existencia, y por ello no faltan voces críticas que rebatan el supuesto pragmatismo de un abordaje del parto tan alejado de las pulsiones intuitivas. Desde este contrafuego ideológico, se argumenta que existen evidencias de que la disminución en la mortalidad materno-infantil no se puede achacar a la aplicación de la tecnología hospitalaria sobre el parto, sino como afirma el Dr. Wagner (1989) —excomisario de la Organización Mundial de la Salud (OMS) en salud reproductiva-, a otras circunstancias como una mejor nutrición, una mayor salud de las mujeres, una disminución de la natalidad y algo muy importante, unas mejores condiciones de la vivienda y la higiene ${ }^{2}$.

\footnotetext{
${ }^{1}$ Tal como apunta Alferedo (1989) la disminución de la mortalidad infantil durante el siglo XX se cifra en un $71 \%$ en los países desarrollados y un $51 \%$ en los subdesarrollados.

${ }^{2}$ Autores como Fausto Dopico (2000) han puesto de relieve que el descenso de la mortalidad materno-fetal se generalizó mucho antes de la aparición de los antibióticos y de cualquier medicamento eficaz contra las infecciones. Como señalan autores como Michael Foucault (1990), Josep Bernabéu y Sonia Contada (1991), a pesar de que la reducción fue atribuida a la medicalización hospitalaria de los partos, parece que en este descenso influyeron más las variables del medio que los avances médicos. Variables como el aumento del nivel educativo que, como muestra el estudio de Elena Robles, Josep Bernabéu y Fernando García Benavides (1996), tuvieron un papel crucial en la disminución de la mortandad, produciéndose una reducción de la mortalidad infantil de entre un $7 \%$ y un $9 \%$ por cada año adicional de escolarización. Otros autores (Gómez 1985) muestran cómo la disminución de la mortalidad infantil en España, durante del siglo XX, se ha debido a las mejoras en la alimentación y en las condiciones de salubridad, mientras que
} 
Pero las perspectivas críticas profundizan aún más en esta trama y diferentes investigaciones (Tew 1985 y 1990; Campbell y Macfarlane 1987; Olsen 1997) apuntan a que el parto en el hospital ha podido incluso aumentar la morbimortalidad de las madres y los bebés, no existiendo pues evidencia de que sea más seguro para las mujeres parir en éste. La conclusión a la que llegan estos estudios es que una intervención obstétrica puntual puede salvar la vida de determinadas mujeres y sus bebés, pero cuando se interviene de forma sistemática en casos de bajo riesgo, esa intervención incrementa considerablemente el peligro de complicación. De esta manera, el parto en casa podría considerarse tan seguro como en el hospital para mujeres de bajo riesgo, pero en el hospital las mujeres de bajo riesgo estarían expuestas a mayores complicaciones durante y después del mismo. Desde esta óptica, el descenso de la mortalidad materno infantil no se ha debido tanto a lo que han hecho los obstetras en el medio hospitalario, sino a los autocuidados que empezaron a practicar las mujeres en sus propias casas (Wagner 1989). Las controversias se han visto avivadas, aun más, por las diferentes estrategias de humanización del parto publicadas en los últimos años por parte de diversos organismos sanitarios oficiales ${ }^{3}$. Se trata de documentos que han apostado por la adopción de patrones de asistencia al nacimiento menos intervencionistas, pidiendo a su vez un mayor esfuerzo investigador en torno a la forma en que se están asistiendo los partos y una revisión de las diferentes tecnologías que se aplican en los mismos.

Existe pues un auténtico debate en torno a la forma en que se está enfocando la asistencia al parto en Occidente. Como en cualquier disputa las posiciones antitéticas, esgrimidas por los que podríamos catalogar como intervencionistas y naturistas del parto, dibujan en cierta medida los extremos del problema, en una sociedad actual que con frecuencia lleva al extremo el principio de los opuestos, en oposición al "justo medio" aristotélico. Las pesquisas intelectuales en torno a este nudo gordiano ya han comenzado, y las espadas dialécticas están en alto, por lo que el esclarecimiento de la propia idiosincrasia de las diferentes representaciones y perspectivas asistenciales parece una labor útil, en aras de la mayor comprensión de nuestra propia sensibilidad.

La compresión de todas estas escaramuzas ideológicas parte, sin duda, de la asunción de que el parto, como cualquier otra realidad social, se construye y configura en clave cultural. Por tanto, las representaciones que existen sobre el mismo no son aleatorias, sino que se originan en una matriz contextual e histórica concreta, al formarse dentro de un marco biosocial producido, tanto por factores biológicos universales, como por factores culturales particulares (Jordan 1993). Estas representaciones ideológicas se instituyen en auténticos paradigmas obstétricos que dibujan el "conjunto de prácticas autorizadas durante un período específico de tiempo" (Kuhn 2005: 10) para dar cobertura a los procesos del nacimiento. Un conjunto de prácticas que han sido agrupadas en diferentes paradigmas ideológicos, que representan a su vez diferentes cosmovisiones del parto, situándose las perspectivas tecnocráticas, típicas de

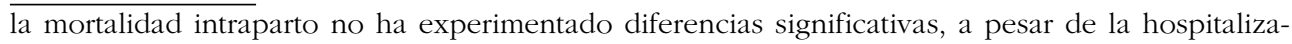
ción de los nacimientos acaecida durante la segunda mitad de siglo.

${ }^{3}$ Entidades oficiales como la Organización Mundial de la Salud (1985, 1996), el Ministerio de Sanidad y Consumo español (2007, 2010), la Sociedad Española de Ginecología y Obstetricia (2008) o la Federación de Asociaciones de Matronas de España (2008) han editado documentos a favor de la desmedicalización y humanización del parto. 
la biomedicina, en el espectro de la medicalización de la asistencia, en contraposición con los enfoques humanizados y holísticos, situados en la búsqueda de una armonía entre las necesidades de las instituciones hospitalarias y las de las propias madres (Davis-Floyd 2001). Estas cosmovisiones se traducen en la práctica clínica en auténticas "culturas del nacimiento" (Montes 2007:10) o "etno-obstetricias" (McClain 1975) ${ }^{4}$, que recogen las diferentes maneras en que cada cultura particular establece los itinerarios asistenciales del mismo. Estos patrones culturales de atención, en consecuencia con las representaciones que los alimentan, cristalizan en diferentes arquetipos asistenciales, como el parto institucionalizado-intervencionista o medicalizado, típico del paradigma tecnocrático, o el parto hospitalario-humanizado y domiciliario, propio de las vertientes humanitaria u holística (Blázquez 2009). Estos patrones se encuentran en continua pugna por la hegemonía ${ }^{5}$ ideológica, siendo en nuestro contexto el parto institucionalizado e intervencionista la modalidad más común, mayoritaria y legitimada desde la segunda mitad del siglo XX (Martin 1989). Estamos ante un patrón asistencial que ha encontrado su justificación clínica en un supuesto pragmatismo epidemiológico convenientemente higienizado de las causas culturales, pero en cuya difusión y asentamiento han influido factores sociológicos curiosamente negados desde las ciencias biomédicas. Estos factores y razones de fondo se exploran en el presente artículo, a través de un análisis documental exhaustivo, con el objetivo de dilucidar las principales características y bases antropológicas que sustentan el modelo.

El análisis documental se centró en el examen de documentos pertenecientes a diversas áreas de conocimiento como la antropología, la sociología, la obstetricia o la matronería, revisándose libros, monografías, tesis doctorales, artículos indexados en bases de datos del ámbito de las ciencias sociales y médicas (Antro Base: Social and Cultural Anthropology, Current Contens, SOC-Antropología social, Periodicals Archive Online - PAO_, Pudmed, Cochrane), declaraciones, estrategias, guías de práctica clínica, programas, protocolos asistenciales, documentos utilizados en centros sanitarios (cartilla maternal, folletos informativos, etc.), e información manejada por las madres no perteneciente a los circuitos formales (información vertida por asociaciones relacionadas con la maternidad a través de páginas Web y folletos no oficiales).

\footnotetext{
${ }^{4}$ Los términos "culturas del nacimiento" o "etno-obstetricias" hacen referencia a las diferentes representaciones simbólicas y praxis derivadas que caracterizan a un determinado abordaje cultural del parto.

${ }^{5}$ Antonio Gramsci introdujo el concepto "hegemonía" y "bloque hegemónico" para referirse a la supremacía cultural que las clases dominantes lograban ejercer sobre las clases sometidas. El concepto ha sido llevado al campo de la "antropología de la medicina" por el antropólogo argentino Eduardo Menéndez, para referirse a la hegemonía que ejerce el sistema médico occidental, al que cataloga con el apelativo de "Modelo Biomédico Hegemónico", sobre otros sistemas médicos que quedan arrinconados y convertidos en subalternos. No es un tema ajeno a lo que aquí se plantea, ya que el modelo de asistencia del parto que en estos momentos se comporta como hegemónico en Occidente es el "parto institucionalizado" que, como no podía ser de otra manera, es el idiosincrásico de la biomedicina occidental.
} 


\section{CARACTERÍSTICAS Y ATRIBUTOS}

El parto hospitalario es una "cultura del nacimiento" que ha sido etnografiada en los últimos años desde diferentes perspectivas, entre ellos pueden citarse análisis como el de Franca Pizzini (1989), que describe las diferentes escenas de medicalización que acontecen en los paritorios italianos, los de Teresa Joaquim (1983), que aborda las creencias y prácticas en torno al embarazo y parto hospitalario en Portugal, o los de Brigitte Jordan (1993), que compara el parto en cuatro países diferentes -México, Holanda, Suecia y EE.UU.- haciendo un análisis crítico del excesivo intervencionismo del parto en EE.UU. Ya en el ámbito español, destacan algunos estudios como los de María Luz Esteban (1992), Yolanda Bodoque (1996), María Jesús Montes (2007), María Isabel Blázquez (2009) o José Manuel Hernández (2011), centrados en los itinerarios asistenciales del parto institucionalizado. Se trata, en definitiva, de toda una serie de etnografías que tratan de dibujar el objeto de estudio y que han permitido caracterizar al parto institucionalizado como una etnoobstetricia vertebrada por tres atributos cardinales: la medicalización de los procesos normales, la obsesión por el control biotecnológico y la organización protocolizada y mecanizada del trabajo ${ }^{6}$. Estamos, pues, ante "un modelo intervencionista e institucionalizado, atendido por profesionales de la medicina especializados" (MSC 2007:16), que cobra vida en la medida en que sus idiosincrasias encuentran el babitus ${ }^{7}$ necesario para su realización. Por ello sus propiedades nos hablan de una doble finalidad, la de mediadoras en la constitución de los escenarios de origen, y la de pilares de cuyo mantenimiento depende, en última instancia, el mantenimiento del sistema. Analicemos, por tanto, cada uno de estos caracteres, deteniéndonos en las implicaciones que tienen para la maternidad.

\section{TENDENCIA A LA MEDiCALIZACiÓN}

El nacimiento no ha escapado a la tendencia medicalizadora (Stoller 1974), y a pesar de ser uno de esos procesos que ni para el sujeto, ni para el clínico, constituyen una enfermedad (Foucault 1990), se ha instituido en un ejemplo paradigmático de ésta (Zola 1981), entendida como una actividad que se ha ido "expandiendo, en años recientes, a muchos problemas que antes no eran considerados como entidades médicas" (Márquez y Menau 2003:47-43), interponiéndose impúdicamente en el flujo natural de las interacciones materno-fetales.

De esta manera, la injerencia en el parto, producida tras su hospitalización en la segunda mitad del siglo XX (Vía 1972; Hernández 2012), ha encontrado su modus

\footnotetext{
${ }^{6}$ Autoras como Nancy Stoller (1974:131-142) o Brigitte Jordan (1993), tras estudiar el parto hospitalario en Estados Unidos, han otorgado diferentes atributos al parto hospitalario, entre los que destacan su tendencia a la medicalización, su abordaje autoritario y asimétrico, su obsesión por el control tecnológico y la fragmentación de los cuidados.

${ }^{7}$ El habitus es uno de los conceptos centrales de la teoría sociológica de Pierre Bourdieu. Por tal podemos entender esquemas de obrar, pensar y sentir asociados a la posición social, que hacen que personas de un entorno social homogéneo tiendan a compartir concepciones y estilos de vida parecidos.
} 
operandi en la progresiva problematización de la reproducción, como instrumento indirecto de control social (Esteban 1996). Las nuevas coordenadas orgánicas han llevado a definir la morfología femenina como tendente a la patologización (De Miguel 1979) y a las mujeres como víctimas en sí mismas por su naturaleza biológica pasiva (Douglas 1996; Burin et al. 1990; Stolcke 2003). Vivimos, pues, una distorsión por la que los ritos alumbratorios son considerados como un periodo liminal o etapa de transición (Imaz 2001), bloqueando, así, el miedo a la incertidumbre, los recursos ancestrales y las capacidades decisorias. Estamos ante una experiencia extra-corporal, ante un desdoblamiento por el que las mujeres viven sus embarazos y partos a través de los especialistas, careciendo sus sensaciones de valor objetivo. Se trata, en definitiva, de un viaje astral, de una apropiación de sus propios sentidos que reviste a los profesionales en expertos del conocimiento de las cosas tal y como ellos las definen, y a las madres en "invalidas, dependientes del médico, para curar 'manifestaciones' propias de su cuerpo" (Juan 1991: 38).

Desde esta perspectiva, la asistencia se construye a través de toda una "cascada de intervenciones" que elevan progresivamente los riesgos (Inch 1982), realidad que es vivida como parte "natural" del proceso, y no como una cosmovisión cuya consecuencia final es la "iatrogenia institucionalizada". Es un escenario que no se apoya, como sería previsible, en las pruebas empíricas del positivismo, sino en la mística de los "metarrelatos" del modernismo, no existiendo, de hecho, ninguna evidencia de las ventajas del intervencionismo sistemático en los partos normales (Thomson 2000). Estamos, pues, ante una representación teatral que genera sentimientos de inseguridad y desconfianza, promoviendo, a su vez, la delegación forzada del poder intuitivo materno al personal sanitario (Narotzky 1995). Se trata, sin más, de un habitus, mediado por el paso a través de diferentes escenas de adaptación durante la estancia en las salas de parto (Pizzini 1989), que garantiza el control de las neófitas por medio de la normalización de su conducta bajo la égida de las nociones de la tecnociencia (Esteban 1992). La justificación del uso de la medicalización indiscriminada en el parto "hay que buscarla, por tanto, en el contexto del ejercicio de control que [sobre la maternidad] ejerce el sistema [hospitalario], de quien se espera que dé solución a unas manifestaciones físicas que, aunque normales, se viven como irregulares y amenazantes" (Montes 2007: 44).

\section{OBSESIÓN POR EL CONTROL BIOTECNOLÓGICO}

Otra de las características que vertebran esta "cultura del nacimiento" es su propensión a significar los partos desde la perspectiva tecnológica. Predisposición, cuyo origen se puede situar en la introducción de la tecnociencia en los alumbramientos, y más concretamente en la creación de los primeros bioinstrumentos aplicados a éstos, como el fórceps o el cloroformo (Narotzky 1995; Hernández 2012). Desde ese momento la tecnología ha ido cobrando cada vez más relevancia en el manejo de la reproducción (Oakley 1984), apoyada, como no, en los discursos de la prevención de riesgos.

Esta obsesión por el control biomecánico de los cuerpos maternos ha encontrado su modus vivendi en la necesidad de aportar datos objetivos sobre las desviaciones 
biológicas del parto. Desviaciones que "la tecnología [posibilita], al penetrar en el cuerpo sacando a la luz señales que son codificadas y significadas a través de los saberes culturalmente establecidos" (Montes 2007: 197). Se trata de un escenario que permite el control biométrico, el "estudio de los rasgos antropométricos susceptibles de ser establecidos y comparados mediante instrumentos y medios técnicos adecuados, posibilitando, así, cuantificar, en términos matemáticos, las características fisiológicas y/o comportamentales" (Fernández 2007: 88) de las madres. Ello ha permitido que por primera vez se imponga "el dato producido por las pruebas técnicas, como ecografías, monitores o analíticas, que es lo real frente a la práctica sanitaria tradicional basada en la clínica y observación del paciente, denostada por el enfoque tecnológico" (Blázquez 2009: 89). Así, la verdadera patología se revela, ahora, a través de los hallazgos físicos detectados por los artilugios mecánicos, orientándose las actuaciones clínicas en relación con este conocimiento "objetivo" (Good 2003) que se centrará, en adelante, en el continente del feto -el útero- mientras las madres se diluyen como un azucarillo.

Los riesgos de estas perspectivas no estriban en la tecnología en sí misma, sino en la reducción de las madres "a un conjunto de datos, [... hasta el punto] de que se conciban a sí mismas bajo el signo de la técnica" (Fernández 2007: 88). Estamos ante "uno de los cortocircuitos teóricos que la biometría podría activar, la simplista, pero seductora confusión, entre lo que es medido, y el ser humano mismo" (Saraceni 2005: 88). Una distorsión que puede conducir a la creación de «un cuerpo cada vez más manipulable a través de la tecnología, una tecnología que se interpone e interrumpe el flujo de las interacciones" (Marchesini 2002: 245). Se trata de una nueva forma de afrontar la maternidad, el parto "biolítico" ${ }^{8}$, cuya representación se sustenta en una simbiosis de las parturientas con las máquinas que las equipara a objetos inertes. El riesgo de la biometría radica, en definitiva, en el hecho de que los expertos dejen de mirar a las gestantes a los ojos, para desplazar su atención hacia un sinfín de artefactos mecánicos que nada entienden de su subjetividad, ni de sus necesidades emocionales, reduciendo así, de forma definitiva, lo que quedaba del homo patiens a simple información.

Esta obcecación por el control biotecnológico parasita a las propias gestantes, que, inducidas por la entelequia de los artefactos, experimentan una "encarnación tecnológica" (Montes 2007:192-195) que les lleva a que sus vivencias ya no dependan de la sensibilidad inmediata, sino de los datos biométricos aportados por los artilugios mecánicos. Así, las percepciones de las madres quedan condicionadas por los frecuentes exámenes que plasman gráficamente su estado, estando sus sensaciones supeditadas a las definiciones biotecnológicas. El proceso se garantiza a través de "una especie de 'catequesis obligatoria' de desorientación, en el curso de la cual las madres son iluminadas sobre la 'vida' en sus entrañas" (Duden 1996: 91). El objetivo de esta catequesis es el establecimiento de una dialéctica sustentada en la desconfianza sobre el discurso sentivo-corporal, que es sustituido por los datos que dictan los resultados de los exámenes de la ciencia empírica. El abuso de la biotecnología tiende así

\footnotetext{
${ }^{8}$ Término acuñado por el periodista y escritor francés Hervé Kempf (1998) para referirse a la simbiosis entre el hombre y la máquina, a la equiparación entre lo vivo y lo mineral que genera una nueva forma de transhumanismo.
} 
a reforzar una visión de la maternidad como enfermiza, hecho que justifica la biointervención y convierte, tanto a las madres como a sus criaturas no nacidas, en víctimas de problemas y enfermedades (Corea et al. 1987).

Estamos, pues, ante un afrontamiento biotecnológico que reafirma la subordinación materna, al transformar un proceso intuitivo en algo que, pese a suceder en y desde el cuerpo de las mujeres, es interpretado y manejado desde el "saber" de los asistentes. Las máquinas sirven, por tanto, para potenciar el poder de la tecnociencia en las definiciones de la salud y la enfermedad, al anular el saber y la autonomía de las mujeres. Desde esta doctrina, éstas tienen que consultar periódicamente a los sanitarios, que son quienes dan explicación de lo que acontece, lo que sucede en sus cuerpos. Ello proporciona un poder próximo a lo mágico-religioso, dando a los expertos un hálito de divinidad derivado de la capacidad de interpretación de los galimatías venidos del más allá de las pantallas instrumentales. No es de extrañar, por tanto, que desde estas posiciones el feto "no tenga más como portavoz a la madre sino al clínico" (Imaz 2002: 4).

El uso de la tecnología se ha convertido, así, en un instrumento social, político y económico (Ginsburg y Rapp 1995). De manera que las tecnologías sirven, también, a intereses socioeconómicos de determinados grupos, teniendo las madres que prescindir de su protagonismo en aras de réditos burocráticos y profesionales (González de Chávez 2001). Y es que tener hijos se ha convertido en algo más que un objetivo de las mujeres, se ha convertido en un proyecto de las sociedades capitalistas, con raíces en políticas eugenésicas, respaldado desde las ciencias clínicas (Rapp 1999). Este control se produce desde la misma concepción, por medio de las técnicas de reproducción artificial, pero también durante el embarazo, cuando los fetos son seleccionados según sus posibilidades productivas a través de técnicas como la ecografía o las "amniocentesis eugenésicas", y por supuesto durante el parto, donde el control biométrico determina la viabilidad de un feto sano y funcional.

Queda, pues, bien patente el riesgo del abuso de la biotecnología en el ámbito de la maternidad. Abuso que, en última instancia, puede propiciar la limitación de la experiencia a la simple biometría, vehiculizándose, así, una "encarnación tecnológica" que asegure la subordinación de las madres a los intereses del poder técnico-estatal.

\section{ORGANIZACIÓN DEL TRABAJO PROTOCOLIZADA Y MECANIZADA}

Igual que ocurría con la medicalización, el parto constituye también un ejemplo paradigmático de protocolización-mecanización de la atención clínica (Zola 1981). Esta rigidez se ha producido como consecuencia de la especialización y fragmentación del cuidado, acaecida tras la hospitalización definitiva de los procesos de salud-enfermedad, y que ha llevado a hacer rutinarios los procesos asistenciales a través de toda una serie de protocolos, procedimientos y pruebas, cuyo objetivo es alcanzar la "uniformización" de los itinerarios clínicos (Esteban 2001). Se ha conseguido, así, dar la "Condición de asistabilidad" al parto, condición que "no depende necesariamente de un substrato biológico o psíquico, sino de la existencia de un conjunto de reglas, necesariamente arbitrarias" (Comelles 1985: 140), y cuyo fin último es garantizar que la madre sea incluida en el recorrido hospitalario estipulado. Desde esta perspectiva 
se han especificado claramente todas "las pautas, controles y pruebas que se recomiendan a lo largo del embarazo y parto, así como las posibles diferencias o cambios que en cada mujer se pueden dar, y cómo y cuál debe ser la actuación en todos los casos" (Montes 2007: 55). Se instituyen, así, los protocolos en "guías" de obligado cumplimiento para los equipos sanitarios, que planifican sus actividades en un orden preestablecido y mecanizado. No ha de extrañar, pues, que los partos estén impregnados de experiencias de sometimiento a innumerables tareas repetitivas, quehaceres que los profesionales deben realizar amparados en la simple justificación de que el protocolo lo establece.

Estamos ante una degeneración ritual por la que los procesos alumbratorios se han implantado de tal manera, que las madres, desde el primer momento del embarazo, se ponen en contacto con los profesionales para que les organicen todo un programa automático de visitas. Secuencia mecanizada que es, además, registrada en documentos escritos, como las cartillas maternales, las historias clínicas o los partogramas, manuscritos que se convierten en auténticos instrumentos fiscalizadores y controladores, al aportar todos los datos sobre el grado de cumplimiento de los itinerarios, convirtiendo, así, a las gestantes en objetos de estrecha vigilancia. La mecanización no sólo afecta a la praxis, sino también a los discursos, ya que en los protocolos se especifica que es necesario "vehicular la información", es decir dar una información preestablecida que evite el libre albedrío informativo de los profesionales. Se trata, en definitiva, de un galimatías burocrático legitimado por los especialistas y por la creencia popular en el poder de la tecnociencia para prevenir y curar nuestras imperfecciones, por ello el enfoque rara vez es cuestionado, colaborando en el proceso hasta las propias madres que acatan y se someten a todas las pruebas, y exploraciones íntimas, que dicte el protocolo.

Los protocolos se convierten, así, en materia de obligado cumplimiento, debiendo asumir las madres de forma vertical, y al margen de sus opiniones, conocimientos o estado clínico, todos los discursos e ideologías institucionales. Discursos que deben interiorizar, dando muestras fehacientes de participación y responsabilidad en su aprendizaje y reproducción. El miedo al riesgo y las connotaciones morales de recriminación que puede conllevar el no cumplimiento de las normas, favorece la manipulación moral. La obligatoriedad en el cumplimento afecta también a los propios profesionales, al convertirse las pautas que dictan estos documentos en una especie de "reglamento formal" que adquiere tintes de ley. Así, su acatamiento se convierte en una responsabilidad que garantiza una atención de "calidad", convirtiéndose los sanitarios en auténticos guardianes del orden establecido, sobre todo en el caso de las profesiones paramédicas, que asumen de forma diligente el papel de controladores de la observancia de las indicaciones?.

La mecanización de la asistencia contribuye también, en parte, a la liberación de las responsabilidades profesionales. En una sociedad en la que las demandas por mala praxis obstétrica se han convertido en una conducta habitual (Cunningham 1998; Martin 1987), llevar a cabo todo lo que dictan los protocolos protege de posibles contra-

\footnotetext{
9 Autores como Sarlet, García y Belando (1996), tras un análisis antropológico de los procesos asistenciales, han concluido que son las profesiones paramédicas las que cumplen el papel de difundir las ideologías biomédicas relacionadas con los itinerarios de atención.
} 
tiempos, justificando unas decisiones clínicas que forman parte del engranaje "normal". De esta manera, aunque son los expertos los que dictan las pautas, no se hacen responsables de lo que en última instancia pueda ocurrir, descargando sistemáticamente las responsabilidades sobre las madres, que deben firmar múltiples consentimientos informados y cumplir sin rechistar los formulismos. Se produce así una conversión de las pacientes en objetos pasivos y sumisos de intervención, en "máquinas corporales" que deben ajustarse a unas prácticas que se aplican como si de un solo cuerpo se tratara. Noción que refuerza la actuación del clínico sobre el cuerpo, al convertirse en una especie de mecánico-técnico que arregla la máquina (Martin 1987).

La consecuencia final es una falta de la individualización asistencial desde la que no se pueden integrar las diferentes necesidades, emociones y opiniones maternas. Estamos ante una rigidez conceptual que parte de "una concepción mecanicista del cuerpo y del hospital como una fábrica de sacar niños" (Montes 2007: 246). Así, el nacimiento transcurre como un proceso rígido, como un trance inflexible dictado por férreos protocolos, cuyo curso se vertebra a través de una "ceremonia ritual" desde la que cobran sentido muchas de las rigideces que forman parte estructural de la asistencia de los procesos reproductivos. Asistimos, pues, a una deshumanización de la asistencia (Hernández 2009) en la que las rutinas, características del parto biotecnológico, "dejan de ser actuaciones puntuales para convertirse en prácticas cotidianas que se normalizan y naturalizan" (Blázquez 2009: 293).

\section{BASES ANTROPOLÓGICAS}

Tras un itinerario asistencial tan alejado de los ritmos e instintos maternales se esconde toda una serie representaciones ideológicas de las que depende, en última instancia, la comprensión de los atributos litúrgicos de esta "etno-obstetricia". Filosofías de fondo que, a pesar del discutido pragmatismo del modelo asistencial, subyacen a la propia dinámica del ritual y nutren las características litúrgicas relatadas en el apartado anterior. Bases sociantropológicas como la biopolítica, u obsesión por el control estatal de la reproducción, que alimentan la tendencia a la medicalización, el posthumanismo, o propensión por significar a las madres a través de la técnica, que sostiene la tendencia a la tecnologización, o el productivismo, o representación del parto como un proceso productivo más, que mantiene la conceptualización del nacimiento en clave de mecanización. Examinemos por separado cada una de estas bases antropológicas con el fin de desentrañar el lado negado del parto institucionalizado, y con el objetivo de esclarecer los auténticos intereses y cosmovisiones que lo sustentan.

\section{Biopolítica}

Cuando hablamos de biopolítica nos referimos a la forma en que las organizaciones nacionales y estatales se han ido introduciendo en el control de los procesos biológicos, con el fin de garantizar ciudadanos útiles y productivos. Para ello "el Estado ha garantizado la hegemonía del poder técnico y profesional de los especialistas, que 
ha cumplido sobre todo funciones de control y legitimación" (Menéndez 1984: 36), imponiéndose así la ciencia "al individuo, enfermo o sano, como un acto de autoridad" (Foucault 1990: 60). De esta manera, la sapiencia clínica se ha ido dotando "de un poder autoritario con funciones normalizadoras que han ido más allá de la existencia de enfermedades" (Foucault 1990: 180), y que responde a los intereses y necesidades de las naciones. Su poder de normativización ha comprendido tanto la enfermedad como la salud, pretendiendo el control de los cuerpos que han sido domesticados según las necesidades del orden social y político (Scheper-Hughes y Lock 1987). Estamos ante una estrategia por la que la profesión hipocrática se ha ido convirtiendo, entre nosotros, "no sólo en un segmento importante de nuestra cultura sino en el dominante, no estando incluso muy lejos de ser él toda la cultura" (Laplantine 1999: 380-381). Hasta el punto de que "ha sido organizada de la misma manera que el clero e investida, en el nivel corporal y de la salud, de los poderes que éste ejerce sobre las almas" (Foucault 2007: 42). Así, se ha asegurado que el control estatal "no se opere simplemente desde la conciencia y la ideología, sino que se ejerza también sobre el propio organismo. Organismo que se ha convertido en una realidad biopolítica, a la vez que [la clínica] se transformaba en un estrategia biopolítica" (Foucault 1990: 125).

Esta tendencia ha afectado de lleno a la reproducción, cuyo control se ha convertido en "una medida política de protección a las criaturas por nacer, puesto que son consideradas como bienes sociales necesarios" (Montes 2007: 116). Por ello, "el sistema sanitario vigila las condiciones de producción, y las de la mujer que aporten datos sobre el hecho de que el feto se desarrolla adecuadamente, y que los embarazos se ajustan a la finalización del proceso" (Blázquez 2009: 95). Aunque suene un poco drástico, se podría decir que estamos ante una nueva explotación de las mujeres con fines de reproducción social (Tabet 1985). Una explotación que las ciencias empíricas han perpetrado a través de la medicalización sistemática de los "úteros maternos", a fin de garantizar unos estándares de producción. Se ha generado, así, una eugenesia cultural apoyada en la inseguridad que producen discursos como el del riesgo y el dolor ${ }^{10}$, que, a su vez, garantizan el acatamiento y sumisión a las pautas estatales y profesionales.

$\mathrm{Y}$ es que "la idea del riesgo en los partos normales ha conducido a dar protagonismo a la enfermedad frente a la salud, dejando de ser la reproducción una experiencia cotidiana de la vida de las mujeres, como ocurría en épocas anteriores" (Blázquez 2009: 101-102), para convertirse en una enfermedad más. Se trata de un camino sin retorno por el que la posibilidad de riesgo ha desestructurado la seguridad cotidiana, haciendo dóciles a las mujeres en manos de los especialistas (Taussing 1995). La situación aún cobra mayor importancia cuando los discursos de las políticas sanitarias que regulan el nacimiento, no contemplan el riesgo cero y cuando, además, los programas de prevención delegan la responsabilidad en las propias "pacientes" (Montiel 1997), que son culpabilizadas de los daños si no acatan las directrices estatales. Así, las estrategias de prevención de riesgos se convierten en instrumentos

\footnotetext{
${ }^{10}$ La antropóloga y comadrona María Jesús Montes (2007: 40-50) hace una interesante reflexión sobre cómo estos dos discursos han difundido la inseguridad en las madres, hasta el punto de que sus recursos naturales quedan anulados, arrojándose como bálsamo redentor al aura de supuesta seguridad que le da el control biomédico del proceso.
} 
políticos de control, al ser todo en la vida susceptible de riesgos. Desde esta perspectiva, el proceso reproductivo es entendido como una situación de especial interés para los Estados, por lo que supone de reemplazo de los individuos, en cuanto a su valor económico como fuerza de trabajo. Todo ello invisibiliza el hecho de que la prevención de los peligros genera, a su vez, nuevos riesgos, desoyendo así los efectos iatrogénicos de la injerencia clínica (Illich 1978).

Otra de las herramientas gnoseológicas que ha garantizado la sumisión materna a la medicalización, ha sido el discurso sobre la abolición del dolor. Disertación que, como cualquier cognición, ha evolucionado paralelamente al proceso cultural y tecnológico, perdiendo, en nuestro contexto, las connotaciones de purificación, expiación del pecado, y fortalecimiento del vínculo madre-hijo, que tenía bajo la ideología cristiana (Roca 1996), para ser concebido ahora, desde las ciencias clínicas, como algo innecesario, inaceptable y devaluado (Winston y Parris 2000). Sinsentido, que redunda en un aumento de la violencia de los rituales alumbratorios (Le Breton 1999), mediando en el tránsito del dolor al sufrimiento que se produce cuando las madres pierden el control, al depender de agentes externos para su alivio (Montes 2007). La consecuencia final es la instauración de un tratamiento estandarizado del dolor, que justifica la medicalización de los partos y que desvaloriza otros aspectos en su reducción, como la autogestión de la mujer u otras técnicas de afrontamiento no biomédicas. Se refuerza así la autoridad del experto y la visión del parto como algo negativo, como un mal del que sólo los técnicos pueden liberar a la mujer, fortaleciendo una actitud pasiva que engrandece la autoridad profesional sobre los "cuerpos grávidos".

Estamos, pues, ante una espiral que se autoalimenta, ante una hélice conceptual por la que la biopolítica — la obsesión del Estado por controlar los "cuerpos gestantes" encuentra su estatus de posibilidad en la delegación del poder en las ciencias clínicas, que ejercen su control, y autoridad, a través de la medicalización sistemática de los partos normales. Injerencias que son justificadas, a su vez, por discursos como el del riesgo y el dolor que, al acrecentar las inseguridades maternas, hacen maleables las voluntades. Se cierra, así, un círculo vicioso que legitima la eugenesis estatal sobre la reproducción, realidad que será negada, como tal, mientras los expertos la avalen.

\section{POSTHUMANISMO}

El posthumanismo es una filosofía que pretende la superación de lo humano a través de la exaltación de la tecnología, como medio de liberación de la persona frente a sus limitaciones y deficiencias físicas y morales (Ballesteros 2007). Estamos ante una corriente ideológica cuyo origen podemos situar en Francis Bacón ${ }^{11}$ y que proclama el ocaso del organismo tal y como lo conocemos (Caronia 2001), despreciando la propia biología corporal (Punzi 2003). La actitud que subyace al posthumanismo «es la del control sobre la naturaleza con que despuntó la ciencia moderna, sólo que ahora

${ }^{11}$ El célebre filósofo y político Francis Bacon recoge en su libro "La nueva Atlántida" (1960) las bases de los postulados posthumanistas, que se fundamentan en el desprecio hacia la naturaleza y lo orgánico. 
extendida a la propia naturaleza humana, con la que se pretende hacer tanteos para averiguar sus posibilidades de mejora" (Ferrer 2007: 156). Se trata de una nueva vuelta de tuerca en ese proceso de abominación de lo humano, de manera que "la noción del 'yo', con que se inauguraba la Modernidad, busca ahora desligarse de los cauces de realización que ofrece su propia [índole] compartida, renunciando a comprenderse desde su identidad biológica específica" (Ferrer 2007: 157).

El posthumanismo se presenta, de esta manera, como un proyecto de mejora del "humanismo" con raíces en ideologías deconstructivistas ${ }^{12}$. Así, si desde el humanismo se "propugnaba que toda [persona], en cuanto tal, tenía una dignidad inalienable y por tanto no podía ser tratada como un medio, ni instrumentalizado en ninguna de sus formas" (Ballesteros 2007: 26), ahora desde el posthumanismo se rechazan las deficiencias físicas y morales, buscando su superación a través de su exaltación biomecánica. El problema se plantea porque "el individuo ha identificado su naturaleza humana con la inseguridad y la vulnerabilidad, por lo que la rechaza y no quiere identificarse con ella" (Viola 2007: 56). Partiendo de estas premisas, "los posthumanistas pretenden utilizar la tecnología para potenciar el cuerpo humano, curándolo, de una vez por todas, de sus debilidades constitutivas" (Saraceni 2007:145). Se trata de una nueva manipulación tecnológico-mecánica, que persigue la simbiosis del individuo con la máquina, el cyborg. Estamos, en definitiva, ante un reduccionismo ontológico que pretende reducir la realidad humana a simple información, ante un proyecto de dudosa eugenesia que cede a las exigencias de la urbanidad.

Estas ideologías posthumanistas han encontrado su caldo de cultivo en el ámbito del nacimiento, redundando en un uso inhumanista de la tecnología que vulnera la dignidad de las madres (Habermans 2002) al cosificarlas, equiparándolas e interpretándolas a través de las máquinas. Se ha producido, así, la paradoja de que han adquirido más importancia los datos biométricos, aportados por la tecnología, que las sensaciones de las propias gestantes. La consecuencia final ha sido el desplazamiento de las miradas hacia los artilugios mecánicos, produciéndose la dilución de las parturientas tras los cortocircuitos tecnológicos que parasitan sus propios organismos. Estamos ante una profanación de los "cuerpos gestantes", que se convierten, desde

${ }^{12}$ En esta corriente posthumanista se sitúan científicos como Norbert Wiener, Julian Huxley, Marvin Minsky o Hans Moravec que pretenden la superación de lo humano a través de la manipulación tecnológico-mecánica. El matemático estadounidense Wiener es considerado como el fundador de la cibernética y a él se deben los deseos de superación del humanismo a través de la "Inteligencia Artificial". Deseos que plasma en libros como Cibernética. El uso bumano de los seres humanos (1969) y El control y la comunicación en animales y máquinas (1985). Por su parte, el biólogo británico Huxley consideraba que esta simbiosis biológica y mecánica (biolítica) constituiría la superación de la humanidad, dando lugar a un nuevo "transhumanismo". Posicionamientos teóricos defendidos en su libro New Bottles for new win (1957). En esta misma línea, el científico estadounidense Minsky se autoproclama un gran defensor de la simbiosis entre el hombre y la máquina, desde posturas que devalúan el cuerpo y el cerebro, demostrando su contrariedad por la subjetividad humana. Ideas defendidas en su libro La sociedad de la mente, la inteligencia bumana a la luz de la inteligencia artificial (1987). Por último, el investigador australiano Moravec desea traducir la subjetividad humana a bits, con el objetivo de que la conciencia humana peregrine desde la carne hasta la máquina, considerando el cuerpo humano como una antigualla y vaticinando la superación del hombre por el "cyborg" en el 2050. Argumentos defendidos en libros como Mind Children (1988). 
ahora, en simples contenedores biológicos que son interpretados mediante los datos aportados por las proyecciones mecánico-tecnológicas. Se corre, así, el riesgo de que las "pacientes" se distancien cada vez más de la experiencia de su entorno y de su propia corporalidad, hasta el punto de que les cueste encontrarse a sí mismas (Barrett 1987). "El riesgo es que la tecnología acabe por disolver todo confín, toda vieja barrera: natural-artificial, mente-cuerpo, fabricado-creado" (Amato 2007: 77), de manera que las parturientas, confundidas por la masa, desaparezcan tras los instrumentos. Se trata del modo en el que las intervenciones biotecnológicas, de la "era posthumanista", modifican la relación con la propia corporeidad (Warwick 2002). Estamos hablando de "prótesis, es decir estructuras artificiales que sustituyen, completan y potencian, en parte o totalmente, una determinada prestación del organismo" (Maldonado 1999) materno. Se trata, en definitiva, de la biolítica que equipara lo vivo a lo mineral (Kempf 1998), surgiendo así un conflicto entre la especial dignidad de las madres y el proceso tecnológico (Arendt 1978). Esta nueva perversión ideológica no es algo menor, sino un modus operandi que lleva a la idolatría tecnológica, a la deshumanización definitiva de los procesos reproductivos (Hernández 2009: 55). Lo que aquí se plantea es la conversión en exploradores de lo desconocido (Giddens 2000), la transformación en apóstatas de la última frontera, el propio "cuerpo gestante", en un afán por superar su propia finitud e imperfección (Saraceni 2005: 55). Se trata de "la consideración [de las madres] como objeto, [lo que] supone una negativa a tratarlas como [seres humanos] con su dignidad" (Marcel 2002: 245-246), la negativa a concebirlas como "fines objetivos, esto es, seres cuya existencia es, en sí misma un fin, y un fin tal que en su lugar no puede ponerse ningún otro fin para el cual debieran ellas servir como medios" (Kant 1992: 64). Estamos pues, ante un circunloquio que reflexiona sobre el hecho de que el binomio maternofetal no se reduzca, desde delirios posthumanistas, a una simple secuencia biométrica legible únicamente desde los artefactos. Reduccionismo que llevaría a concebir la tecnología más allá de sus usos lícitos, para convertirla en una nueva religión (Noble 1999).

\section{PRODUCTIVISMO}

El productivismo puede definirse como una ética en la que el trabajo cumple un papel crucial, al expresar la primacía de la industria y la producción en la sociedad moderna (Giddens 1994). Desde esta perspectiva la productividad medible es el último fin de la organización humana, entendiendo que la mayor producción posible es necesariamente buena para la sociedad, por lo que el trabajo debe organizarse en pos de este objetivo. El origen de esta filosofía hay que situarlo en las reglas del juego capitalista, convirtiéndose, en nuestros días, en una característica dominante que vértebra todos los discursos sociales (Climent 1999).

Uno de los discursos más afectado por esta filosofía ha sido el de la salud, hasta el punto de que se ha articulado toda la atención sanitaria en torno a ella. Así, los itinerarios asistenciales han sido organizados como un trabajo más, en el que el tiempo, el coste y el rendimiento se han convertido en elementos centrales. Ello ha propiciado que los profesionales estén más preocupados por el cumplimiento de los objetivos, reflejados en protocolos, que por el tipo de atención prestada. De manera que 
el hospital se ha convertido en una especie de fábrica, donde todos los esfuerzos e intereses residen en el producto final, la salud, olvidando el proceso. Estamos, pues, ante una "crisis de la clínica" en la que se economiza el encuentro clínico-paciente, relación que se articula en torno a los resultados de las pruebas y exámenes.

Saturado por estas tendencias, no es de extrañar que se haya organizado todo el trabajo hospitalario en base a las leyes de la oferta y la demanda, transformándose la evaluación económica de las actuaciones sanitarias, tanto en términos de efectividad, consecución de los objetivos, como de eficiencia, en lo prioritario, por lo que el logro de los fines, con el menor gasto posible, ha cobrado protagonismo (Oliva et al. 2008). Así, la eficacia y el control de los costes se han convertido en los aspectos básicos que determinan la toma de decisiones de los sistemas sanitarios públicos, y fruto de ello los procesos asistenciales han sido clasificados en los llamados Grupos de Diagnósticos Relacionados (GRD), etiquetas que, utilizando variables demográficas y de diagnóstico para el cálculo de los costes, han convertido a la salud en un producto estandarizado que hay que ofertar al menor coste y bajo el criterio de equidad. La consecuencia directa ha sido la protocolización-mecanización de la atención sanitaria, ya que la estandarización de la asistencia permite el cálculo y control de los gastos, asegurando además el suministro ecuánime de los mismos.

Procesos como el embarazo, el parto o el puerperio no han escapado a esta lógica productivista y, a pesar de que durante mucho tiempo habían permanecido al margen del proceso capitalista (Stoller 1974; Martin 1987), tras su traslado al hospital han entrado de lleno en éste. De esta manera la asistencia se ha ido instaurando de una forma taylorista ${ }^{13}$, en el sentido de que la organización científica de la misma ha empezado a estar basada en el cálculo y ajuste de los tiempos, que permiten obtener el mejor producto final, a los mejores costes y con los máximos beneficios de producción. Tal traslación ha favorecido que la reproducción, al enmarcarse dentro del modelo clínico, "se haya transformado en un proceso de producción más, aunque su finalidad sea producir salud y prevenir enfermedades, a la vez, claro, que se producen personas" (Blázquez 2009: 94). A partir de estas nociones se ha tomado en cuenta la duración de las diferentes fases del parto como un valor predictivo. De manera que cuando los tiempos aumentan son percibidos como un indicador de riesgo que refleja la naturaleza defectuosa de las mujeres, justificándose, así, la intervención sobre los cuerpos de las gestantes con el objetivo de adecuarlos a la duración establecida como "normal". Los tiempos se han constituido, de esta manera, en un elemento central en la definición de la normalidad de los partos, convirtiéndose en un instrumento que guía la actuación profesional y sirve para identificar las desviaciones.

Esta filosofía productivista queda reflejada en los diferentes protocolos de asistencia al parto normal, en los que se especifica que "la falta de progreso y detención del descenso implican mal pronóstico, por lo que en estos casos [se debe] extremar

\footnotetext{
${ }^{13}$ El término taylorismo es un derivado del nombre del ingeniero mecánico y economista estadounidense Frederick Winslow Taylor. Hace referencia a la organización del trabajo caracterizada por la división, y asignación de tiempos, a las distintas tareas del proceso de producción. Fue un método de organización industrial cuyo fin era aumentar la productividad, evitando el control del obrero sobre los tiempos de producción. Se trata de una organización laboral íntimamente unida con la producción en cadena.
} 
la vigilancia fetal y replantearse la actuación obstétrica" (SEGO 2008: 7). El desajuste con los tiempos estándar conduce a una mayor intervención profesional, justificando la realización de cesáreas o partos instrumentados, mediante fórceps o ventosas, con el objetivo de favorecer que los nacimientos acontezcan dentro de los tiempos estipulados como "normales". El imperio capitalista llega hasta el punto de que el trance fabril es acelerado a través de intervenciones como romper la bolsa de aguas y administrar determinados fármacos, como oxitocina o prostaglandinas vaginales, ajustándose, así, la cadena de producción de niños a la presión asistencial. De esta manera, si ésta aumenta los profesionales siempre pueden acelerar los partos para que acaben antes, a pesar de que ello suponga un intervencionismo no justificado, o incluso entre en contradicción con las expectativas de las propias mujeres. De la misma manera, una vez obtenido el bebé, el número de intervenciones disminuye, ya que desde perspectivas capitalistas se prefiere que sea la mujer la que se autogestione, disminuyendo así la asistencia. Lógicamente una vez conseguido el producto no se quiere reinvertir tiempo y dinero en él.

Otro de los aspectos que refleja la filosofía productivista, propia de esta "etnoobstetricia", es la construcción ideológica de la ruta asistencial en torno a "la centralidad del producto: el embrión, feto o criatura" (Blázquez 2009: 219). Así, lo que realmente importa es la obtención de un producto apto, por lo que el interés se centra en el control y medición del número de partos realizados, como si de un almacén de stock se tratase. Desde esta perspectiva, el feto se convierte en un paciente más con el que los sanitarios establecen diálogos a través de la ecografía o el registro cardiaco, sin mediación alguna de la mujer (Rapp 1999), vertebrándose todo el proceso en torno al bienestar fetal. Las criaturas y los costes económicos se instituyen, pues, en los ejes de la asistencia, determinando, una vez nacido el bebé, e independientemente de las demandas y necesidades maternas, la clausura del itinerario asistencial bajo criterios de coste-efectividad.

Estamos, pues, ante una endogamia ideológica en la que se espera de las madres "que ocupen su lugar en una 'cadena de producción', que acepten las pautas y cumplan con el rol esperado; lo contrario supondrá que sus profesionales las tengan que convocar, que convencer, que 'obligar' a ello" (Blázquez 2009: 232). Se trata, en definitiva, de una concepción mecánica del cuerpo en la que "la dinámica de la organización del trabajo fabril no puede ser interrumpida. La rueda del sistema tiene unos engranajes preestablecidos para su funcionamiento, y [las madres] deben aprenderlos" (Montes 2007: 146). Desde esta realidad, "la política de gestión se automatiza, hasta el punto de que puede escapar al control de los trabajadores que se convierten en simples ejecutores" (Castel 1986: 219) del sistema. Queda así patente la tradición de la clínica de suministrar cuidados a las parturientas como si de una cinta manufacturera se tratase (Le Breton 1994: 55); una organización, claro está, íntimamente unida a la lógica productivista.

\section{CONCLUSIONES}

Tras la exploración del fenómeno de estudio queda patente que ciertas bases antropológicas están determinando sus características cardinales. Tendencias ideoló- 
gicas como la biopolítica que, como diría Foucault, encuentran su caldo de cultivo en un intervencionismo estatal que pretende ahora domesticar los cuerpos femeninos, en aras de un control de la reproducción con tintes eugenésicos. Los engranajes del modernismo han contaminado pues, a través de la injerencia obsesiva de la tecnociencia, las propias vías de comunicación de los úteros maternos. De la misma manera, los delirios posthumanistas han minado, en el sentido apuntado por Habermans, la propia dignidad de las madres, al representarlas como entes sometidos a los intereses procreadores y no como fines en sí mismos. Se trata del ocaso de lo orgánico, propugnado en su tiempo por Francis Bacon, del delirio de la biometría desde el que la sensibilidad materna ha sido despreciada y sustituida por los fríos datos que dictan los artilugios. Lo importante es el control biotecnológico de los «úteros gestantes", disolviéndose las madres cual frágiles castillos de arena. A todo ello hay que sumar los infundíos del productivismo, que ha encontrado, en contra de lo que hubiera previsto el mismo Adan Smith, su filón de oro en los proceso alumbratorios. De manera que las dinámicas de las cadenas de producción se han instalado en los mismos paritorios, donde las mujeres son asistidas según los intereses reproductivos. Estamos ante el "parto cosmopolita", en el que los diseños tayloristas han convertido a las madres en objetos pasivos y sumisos de una cadena de fabricación de bebés, al estilo más Henry Ford.

En este hábitat ideológico, no ha de extrañar que los partos de la urbanidad estén vertebrados por características como la medicalización, la biotecnologización y la mecanización de la asistencia. Se ha convertido pues, desde esta "etno-obstetricia", al nacimiento en un proceso de consumo más, que alcanza su significación a través de la medicalización de los cuerpos maternos. Se trata de una adulteración que, de otra manera, garantiza el control de la corporalidad de las propias madres, que sometidas a la dictadura de la tecnología esperan que los expertos les dicten las pautas a seguir dentro del ritual. La fragmentación del cuidado ha encontrado en los ceremoniales modernistas su modus operandi, por lo que el protocolo no deja lugar a las improvisaciones intuitivas de las propias madres. Así, durante la liturgia asistencial, se instaura, bajo los discursos del riesgo y del dolor, una dinámica intervencionista en los partos normales por la que las pacientes son abordadas fundamentalmente desde su dimensión biológica, centrándose los asistentes en el control tecnológico de los parámetros físicos del proceso. Esta obsesión por la tecnología y las dimensiones físicas, hace que las madres pierdan su condición de sujeto para convertirse en objeto de intervención. El ritual se encuentra, de esta manera, protocolarizado, adaptándose las praxis a modelos estándar que se aplican de rutina a todas las mujeres. La consecuencia final es la conversión de las mujeres en vehículos pasivos del feto, principal sujeto de interés, formando parte de una cadena de producción ritualizada, cuyas representaciones alimentan, y dan continuidad, a una realidad cultural muy difícil de cambiar mientras haya especialistas que la acrediten.

Estamos, en definitiva, ante un ocaso de la normalidad terciado por la invasión doctrinal de las representaciones de los "cuerpos grávidos", ante un ritual posthumanista que ha depositado en la presunta practicidad de la tecnociencia todas sus expectativas. Un supuesto pragmatismo que supone el último contrafuego ideológico para su mantenimiento, por lo que los expertos, como esclavos del empirismo, intentan fundamentar la totalidad de su praxis desde un aséptico biologicismo, heredero del dua- 
lismo cartesiano. Sin embargo, el pragmatismo de esta "cultura del nacimiento" se desintegra al ser contrastado con las evidencias científicas, apareciendo de inmediato los efectos iatrogénicos de la cascada de intervenciones que la caracteriza. Pese a ello, los positivistas de la tecnociencia, siguen aferrándose a esta quimera, conscientes de que no pueden reconocer que en los rituales obstétricos de la modernidad hay más de cultural, de bases socioantropológicas de fondo, que de practicidad real. Se trata de la parte negada del parto institucionalizado, de una realidad que seguirán obviando, aunque ello suponga ignorar determinados estudios que revelan lo contrario, quedando así claro, tal como ya apuntó Imre Lakatos, que la ciencia no es más que un proyecto de investigación científica que intenta satisfacer los "tics" de cada época concreta, en este caso los de la "era posthumanista".

\section{BIBLIOGRAFÍA CITADA}

Alferedo, E. L. 1989. "Mortalidad infantil: situación y logros". Revista de Divulgación Científica y Tecnológica de la Asociación Ciencia Hoy [Revista electrónica], 1 (2). Disponible en: http://www.cienciahoy.org.ar/hoy02/mortalidad.htm (Acceso 1-22014).

Amato, A. C. 2007. "El cuerpo glorioso, entre representación y experimentación", en Ballesteros, J. y Fernández, E. (coords.), Biotecnología y Posthumanismo: 63-80. Navarra: Aranzadi.

Arendt, H. 1978. La vida del espiritu. Madrid: CEC.

Bacon, F. 1960. La nueva Atlántida. Madrid: Aguilar.

Ballesteros, J. 2007. "Biotecnología, Biolítica y Posthumanismo", en Ballesteros, J. y Fernández, E. (coords.), Biotecnología y Posthumanismo: 21-46. Navarra: Aranzadi.

Barrett, W. 1987. La morte dell'anima. Da cartesio al computer. Roma-Bari: Laterza.

Bernabéu, J. y Contada, S. 1991. "Antecedentes históricos del proceso de socialización de la enfermería española. Desarrollo normativo (1904/1935)". Enfermería Científica 111: 28-36.

Blázquez, M. 2009. Ideologías y prácticas de género en la atención sanitaria del embarazo, parto y puerperio: el caso del área 12 de la Comunidad de Madrid. Tesis Doctoral, Universidad d Rovira i Virgili, Tarragona.

Bodoque, Y. 1996. Discursos y prácticas sobre sexualidad y reproducción. Tesis doctora. Tarragona: Universitat Rovira y Virgili.

Burin, M.; Moncar, E. y Velázquez, S. 1990. El malestar de las mujeres. La tranquilidad recetada. Barcelona: Paidós.

Campbell, R. y Macfarlane, A. 1987. Where to be born? Oxford: Nacional Perinatology Epidemiology Unit.

Caronia, A. 2001. Il cyborg. Saggio sull'uomo artificiale. Milán: Shake.

Castel, R. 1986. "De la peligrosidad al riesgo", en Álvarez-Uría, V. (ed.), Materiales de sociología critica: 219-243. Madrid: La Piqueta.

Climent, V. 1999. Producción y crisis ecológica. Los agentes sociales ante la problemática medioambiental. Barcelona: Edicions Universitat de Barcelona.

Comelles, J. Ma. 1985. "Sociedad, salud y enfermedad: los procesos asistenciales". Jano 655: 71-83. Reimpresión en Trabajo Social y Salud 29, 1997. 
Corea, G. et al. 1987. Man made Women: How Reproductive Technologies Affect Women. Bloomington: Indiana University Press.

Cunningham, G. 1998. Williams Obstetricia. Buenos Aires: Panamericana (20aㅡ ed.).

Davis-Floyd, R. 2001. "The technocratic, humanistic, and holistic paradigms of child-birth". International Journalof Gynecology \& Obstetrics 75 (1): 5-23.

De Miguel, J. 1979. El mito de la inmaculada concepción. Barcelona: Anagrama.

Dopico, F. 2000. "Ganando espacios de libertad. La mujer en los comienzos de la transición demográfica en España", en Duby, J. y Perrot, F. (dirs.), Historia de las mujeres. El siglo XIX: 596-611. Madrid: Taurus.

Douglas, M. 1996. La aceptabilidad del riesgo según las ciencias sociales. Barcelona: Paidós.

Duden, B. 1996. "El concepto de vida: un ídolo moderno y una amenaza para las mujeres embarazadas". Douda 11: 79-96.

Esteban, M. L. 1992. Actitudes y percepciones de las mujeres respecto a su salud reproductiva y sexual. Necesidades de salud percibidas por las mujeres y respuesta del sistema sanitario. Tesis doctoral. Barcelona: Universidad de Barcelona.

Esteban, M. L. 1996. "Relaciones entre feminismo y sistema médico-científico", en Ortiz, T. (ed.), Mujeres de ciencia: 143-184. Granada: Universidad de Granada, Seminario de Estudios de la mujer.

Esteban, M. L. 2001. "El género como categoría analítica. Revisiones y aplicaciones a la salud", en Miqueo et al. (comps.), Perspectivas de género en salud. Fundamentos científicos y socioprofesionales de diferencias sexuales no previstas: 25-51. Madrid: Minerva.

Federación de Asociaciones de Matronas de España, FAME. 2008. Documento de iniciativa a favor del parto normal. Madrid: Autor.

Fernández, E. 2007. "Crítica filosófica del posthumanismo: Gabriel Marcel", en Ballesteros, J. y Fernández, E. (coords.), Biotecnología y Posthumanismo: 81-108. Navarra: Aranzadi.

Ferrer, U. 2007. "Posthumanismo y dignidad de la especie humana", en Ballesteros, J. y Fernández, E. (coords.), Biotecnología y Posthumanismo: 153-170. Navarra: Aranzadi.

Foucault, M. 1990. La vida de los hombres infames. Madrid: La Piqueta.

Foucault, M. 2007. El nacimiento de la clínica, una arqueología de la mirada médica. Madrid: Siglo XXI.

González de Chávez, A. 2001. «Preparación para el parto versus preparación para la maternidad y paternidad", en Género y salud: pp. 24-31. I Jornadas de la Red de Médicas y Profesionales de la Salud. Madrid: Instituto de la Mujer.

Giddens, A. 1994. Consecuencias de la modernidad. Madrid. Alianza.

Giddens, A. 2000. Un mundo desbocado. Los efectos de la globalización en nuestras vidas. Barcelona: Paidós.

Ginsburg, F. y Rapp, R. (eds.). 1995. Conceiving the New World Order: The Global Politics of Reproduction. Berkeley: University of California Press.

Gómez, R. 1985. "El descenso de la mortalidad infantil en Madrid, 1900-1970". Revista Española de Investigaciones Sociológicas 32: 101-139.

Good, B. J. 2003. Medicina, racionalidad y experiencia. Una perspectiva antropológica. Barcelona: Bellaterra. 
Habermans, J. 2002. El futuro de la naturaleza bumana. ¿Hacia una eugenesis liberal? Barcelona: Paidós.

Hernández, J. M. 2009. La deshumanización de la maternidad. Eva expulsada del paraíso. Murcia: Editorial Diego Marín.

Hernández, J. M. 2011. El parto hospitalario e intervencionista. Una construcción social de la maternidad. Tesis doctoral. Murcia: Universidad de Murcia.

Hernández, J. M. 2012. Historia de las matronas. Evolución de la ciencia y el arte de la partería. Murcia: Editorial Diego Marín.

Huntingford, P. 1978. Obstetric Practice: Past, Present and Future. In Place of Birth. Oxford: Oxford University Press.

Huxley, J. 1957. New Bottles for new win. Londres: Chatto \& Windus.

Illich, I. 1978. Némesis Médica. México: Joaquín Mortiz.

Imaz, E. 2001. «Mujeres gestantes, madres en gestación: Metáforas de un cuerpo fronterizo". Política y Sociedad 36: 97-111.

Imaz, E. 2002. "Tres lecturas del cuerpo materno. Antojos, anemias y ecografías", IX Congreso de Antropología FAAEE. Simposio 6: Cultura, poder y salud. Soporte CD.

Inch, S. 1982. Birthrights. Londres: Green Print.

Joaquim, T. 1983. Dar à luz. Ensaio sobre as práticas e crenças de gravidez, parto e pós-parto em Portugal. Lisboa: Dom Quixote.

Jordan, B. 1993. Birth in four cultures. A Crosscultural Investigation of Childbirth in Yucatan, Holland, Sweden and the United States. Montreal: Eden Press Women's Publications.

Juan, M. 1991. "Crear el nacimiento: la medicalización de los conflictos en la reproducción". Revista Española de Investigaciones Sociológicas 53: 29-51.

Kant, I. 1992. Fundamentación de la metafísica de las costumbres. París: Real Sociedad Económica Matritense de Amigos de París.

Kempf, H. 1998. La revolution bolitique: Humains Artificiels et Machines Animées. París: Albin Michel.

Kuhn T. S. 2005. La estructura de las revoluciones cientificas. Madrid: FCE.

Laplantine, F. 1999. Antropología de la enfermedad. Buenos Aires: Ediciones del Sol.

Le Breton, D. 1994. "Lo imaginario del cuerpo en la tecnociencia". Revista Española de Investigaciones Sociológicas 68: 197-210.

Le Breton, D. 1999. Antropología del dolor. Barcelona: Seix Barral.

Maldonado, T. 1999. Crítica della ragione informática. Milán: Feltrinelli.

Marcel, G. 2002. Obras selectas. Madrid: BAC.

Marchesini, R. 2002. Post-human. Verso nuovi modelli di esistenza. Turín: Bollati Boringhieri.

Márquez, S. y Menau, R. 2003. "La medicalización de la vida y sus protagonistas". Gestión clínica y sanitaria 5 (2): 47-43.

Martin, E. 1987. The Woman in the Body. Boston: Beacon Press.

MacClain. 1975. "Ethno-obstetrics in Ajijia". Anthropological Quarterly 48 (1).

Menéndez, E. L. 1984. Hacia una práctica médica alternativa. Hegemonía y autoatención (gestión) en salud. México: Cuadernos de la Casa Chata, 86.

Ministerio de Sanidad y Consumo, MSC. 2007. Estrategia para la atención al parto normal. Madrid: Autor.

Ministerio de Sanidad y Política Social, MSPS. 2010. Guía de Práctica Clínica sobre la atención al parto normal. Madrid: Autor. 
Minsky, M. 1987. La sociedad de la mente, la inteligencia bumana a la luz de la inteligencia artificial. Buenos Aires: Galápago.

Montes, M. J. 2007. Las culturas del nacimiento. Representaciones y prácticas de las mujeres gestantes, comadronas y médicos. Tesis doctoral, Universitat Rovira i Virgili, Tarragona.

Montiel, L. 1997. "Las trampas de la prevención: peligros ocultos en un discurso médico hegemónico", en Montiel, L. y Porras, L. (coords.), De la responsabilidad individual a la culpabilización de la víctima: 253-265. Madrid: Doce Calles.

Moravec, H. 1988. Mind Children. Cambridge: Harvard University Press.

Narotzky, S. 1995. Mujer, mujeres, género. Una aproximación crítica al estudio de las mujeres en las ciencias sociales. Madrid: CSIC.

Noble, D. 1999. La religión de la tecnología. Barcelona: Paidós.

Oakley, A. 1984. The Captured Womb: A History of the Medical Care of Pregnant Women. Oxford: Basil Bernstein.

Oliva, J; Antoñanzas, F. y Rivero-Arias, O. 2008. "Evaluación económica y toma de decisiones en salud. El papel de la evaluación económica en la adopción y la difusión de tecnologías sanitarias". Gaceta Sanitaria 22 (Supl 1): 137-142.

Olsen, O. 1997. "Meta-analysis of the safety of Home Birth. Birth". Issues in Perinatal Care 24 (1): 4-13.

Organización Mundial de la Salud, OMS. 1985. Declaración de Fortaleza. Tecnología apropiada para el parto. Ginebra: Autor.

Organización Mundial de la Salud, OMS. 1996. Cuidados en el parto normal: una guía práctica. Ginebra: Autor.

Pizzini, F. 1989. "The expectant mother as patient: a research study in Italian maternity wards". Health Promotión 4 (1): 1-10.

Press, N. y Browner, C. 1997. "Why women say yes to prenatal diagnosis". Social Science and Medicine 45 (7): 979-989.

Punzi, A. 2003. Lórdine giuridico delle machine. Turín: Giappichelli.

Rapp, R. 1999. Testing women, testing the fetus. The social impact of Amniocentesis in America. Londres: Routledge.

Robles, E; Bernabéu, J. y García Benavides, F. 1996. "La transición sanitaria, una visión conceptual». Boletín de la Asociación de Demografía Histórica XIV (1): 117-144.

Roca, J. 1996. De la pureza a la maternidad. La construcción del género femenino en la post-guerra española. Madrid: Ministerio de Educación y Cultura.

Saraceni, G. 2005. "Cyborg, post-umanesimo e biometría". L'Arco di Giano 45: 75-92.

Saraceni, G. 2007. «El cuerpo del delito. Reflexiones jurídico-filosóficas sobre el posthumanismo", en Ballesteros, J. y Fernández, E. (coords.), Biotecnología y Posthumanismo: 139-152. Navarra: Aranzadi.

Sarlet, A. M; García, A. y Belando, M. R. 1996. Educación para la salud: una perspectiva antropológica. Valencia: Nau Llibres.

Scheper-Hughes, N. y Lock, M. 1987. "The mindful body: a prolegomenon to future work in Medical Anthropology». Medical Anthropology Quarterly 1 (1): 6-41.

Sociedad Española de Obstetricia Y Ginecología, SEGO. 2008. Recomendaciones sobre la asistencia al parto normal. Madrid: Autor.

Stolcke, V. 2003. "La mujer es puro cuento: la cultura del género". Quaderns de l'Institut Catalá d'Antropologia 19: 69-95. 
Stoller, N. 1974. Forced Labor: Maternity Care in the United States. Oxford: Pergamon. Tabet, P. 1985. "Fertilité naturelle, reproduction force", en: N.C. Mathieu (ed.) L'Arraisonnement des femmes. Essais en anthropologie des sexes: 61-146. Paris: Editions de l'Ecole des hautes Etudes en Sciences Sociales.

Taussing, M. 1995. Un gigante en convulsiones. El mundo bumano como un sistema nervioso en emergencia permanente. Barcelona: Gedisa.

Tew, M. 1985. "Place of birth and perinatal mortality". Journal of the Royal College General Practitioner 35: 390-394.

Tew, M. 1990. Safer Childbirth: a Critical History of Maternity Care. Nueva York: Oxford University Press.

Thomson, A. 2000. “Existe la evidencia científica para la medicalización de los cuidados maternales?». MIDIRS, Midwifery Digest 10 (4): 416-420.

Towler, J. y Bramall, J. 1997. Comadronas en la bistoria y en la sociedad. Barcelona: Masson.

Tweedie, J. 1974. "Polished Delivery”. The Guardian, 13-10-1974.

Vélez, A.; Palacios, M.; Ruiz Sternberg, A. M. y Ruiz Sternberg, J. E. 2005. Lecciones de historia del dolor. Bogotá: Centro Editorial Universidad del Rosario.

Vía, R. 1972. Com neixen els catalans. Barcelona: Club editor El Pi de les tres Branques.

Viola, F. 2007. "La defensa de la persona humana en la era tecnológica", en Ballesteros, J. y Fernández, E. (coords.), Biotecnología y Posthumanismo: 47-62. Navarra: Aranzadi.

Wagner, M. 1989. "Nacer en Europa, realidad y perspectivas". I Jornadas Mediterráneas de Embarazo y Parto, Valencia, febrero (sin publicar).

Warwick, K. 2002. I, cyborg. Londres: Century Books.

Wiener, N. 1969. Cibernética. El uso bumano de los seres humanos. Buenos Aires: Sudamérica.

Wiener, N. 1985. El control y la comunicación en animales y máquinas. Barcelona: Tusquets.

Winston C. V. y Parris. L. 2000. "Historia de la medicina del dolor", en Raj, P. P., Tratamiento Práctico del Dolor: 1-10. Madrid: Harcourt.

Zola, I. K. 1981. "El culto a la salud y la medicina inhabilitante", en Illich, I. (ed.), Profesiones inhabilitantes: 37-59. Madrid: Blumen

Fecha de recepción: 8 de abril de 2014

Fecha de aceptación: 25 de septiembre de 2014 